\title{
Femtosecond optical parametric oscillator based on periodically poled $\mathrm{KTiOPO} 4$
}

Article in Optics Letters · February 1998

DOI: $10.1364 /$ OL.23.000061 $\cdot$ Source: PubMed

CITATIONS

49

5 authors, including:

W.P. Risk

IBM

120 PUBLICATIONS 3,391 CITATIONS
READS

23

SEE PROFILE

All content following this page was uploaded by W.P. Risk on 11 August 2015. 


\title{
Femtosecond optical parametric oscillator based on periodically poled $\mathrm{KTiOPO}_{4}$
}

\author{
Tolga Kartaloğlu, Kahraman G. Köprülü, and Orhan Aytür \\ Department of Electrical and Electronics Engineering, Bilkent University, TR-06533 Bilkent, Ankara, Turkey \\ Michael Sundheimer \\ Department of Physics, Koç University, TR-80860 Istanbul, Turkey
}

William P. Risk

IBM Almaden Research Center, 650 Harry Road, San Jose, California 95120

Received July 7, 1997

\begin{abstract}
We report a femtosecond optical parametric oscillator based on a periodically poled $\mathrm{KTiOPO}_{4}$ crystal for which quasi-phase matching is achieved with a $24-\mu \mathrm{m}$ poling period. The singly resonant parametric oscillator, synchronously pumped by a Ti:sapphire laser at a wavelength of $758 \mathrm{~nm}$, generates a signal at $1200 \mathrm{~nm}$ and an idler at $2060 \mathrm{~nm}$. The maximum signal power conversion efficiency of the device is $22 \%$ with a pump depletion of $69 \%$. We tune the signal wavelength over a $200-\mathrm{nm}$ band by changing the cavity length. In addition, pump wavelength tuning provides output tunability in the 1000-1235-nm range. (c) 1998 Optical Society of America OCIS codes: $190.0190,190.2620,190.4970$.
\end{abstract}

Frequency conversion using optical parametric oscillators (OPO's) is a widely used technique for extending the wavelength range of laser systems. Several OPO's have been demonstrated that use a variety of nonlinear materials and pump sources. ${ }^{1}$ Phase matching between the interacting OPO fields is traditionally achieved by use of the natural birefringence of the nonlinear material. In recent years quasi-phase matching, a technique that relies on periodic domain inversion inside the nonlinear material, has emerged as a promising alternative to birefringent phase matching. The domain grating is usually obtained by periodic electric field poling of the nonlinear material. ${ }^{2}$ A number of OPO's based on periodically poled lithium niobate with continuouswave, $Q$-switched, and short-pulse pumping were recently demonstrated ${ }^{3-5}$ indicating a great potential for quasi-phase-matched nonlinear crystals for use in broadly tunable laser systems.

The technology for electric-field poling of lithium niobate has quickly matured relative to that of other ferroelectric materials, primarily because of the wide availability of high-quality and inexpensive crystal substrates. One disadvantage of using periodically poled lithium niobate is that its large coercive field of $\sim 21 \mathrm{kV} / \mathrm{mm}$ limits the poled crystal thickness to approximately $0.5 \mathrm{~mm}$. Another significant disadvantage is the presence of photorefractive damage. Although the photorefractive damage threshold is reduced in periodically poled lithium niobate compared with bulk lithium niobate ${ }^{6}$ satisfactory operation still requires heating the crystal in a temperature-controlled oven. Another material that has much promise for periodically poled nonlinear optics applications is $\mathrm{KTiOPO}_{4}$ (KTP). KTP has a much higher photorefractive damage threshold than lithium niobate, permitting room-temperature operation without temperature control. The much lower coercive field of $\sim 2 \mathrm{kV} / \mathrm{mm}$ permits successful electric-field poling on samples of at least 1-mm thickness. ${ }^{7}$ Many recent advances have been made in electric-field poling of KTP, indicating that periodically poled KTP may become the material of choice for many frequency conversion applications. 8,9

In this Letter we report a room-temperature femtosecond OPO that is based on a periodically poled KTP (PP KTP) crystal. Our OPO is synchronously pumped by a mode-locked Ti:sapphire laser operating at a wavelength of $758 \mathrm{~nm}$. Quasi-phase matching is achieved with a $24-\mu \mathrm{m}$ poling period in the PP KTP crystal, resulting in a signal wavelength of $1200 \mathrm{~nm}$ and an idler wavelength of $2060 \mathrm{~nm}$. One can tune the signal output in a $200-\mathrm{nm}$ range by changing the cavity length. To our knowledge, this is the first reported demonstration of quasi-phase-matched optical parametric oscillation with PP KTP.

In our experiments we use a $z$-cut 1 -mm-thick 8-mm-long hydrothermally grown KTP crystal. We achieve periodic domain inversion in the crystal by applying an electric field along the $z$ axis (1-mm dimension) with a $24-\mu \mathrm{m}$ period electrode. ${ }^{7}$ The resulting domain grating occupies the central $7 \mathrm{~mm}$ of the crystal. The orientation of the grating with respect to the polished crystal surfaces cannot be controlled accurately, and there may be an angle of a few degrees between these surfaces and the grating. The polished crystal surfaces do not have any antireflection coatings.

A mode-locked Ti:sapphire laser provides the pump beam to the OPO at a wavelength of $758 \mathrm{~nm}$. The pump pulses are $215 \mathrm{fs}$ in duration (approximately 1.5 times longer than being transform limited) and 
have a repetition rate of $76 \mathrm{MHz}$. We constructed a ring cavity consisting of four mirrors, as shown in Fig. 1. M1 and M2 are 150-mm radius-of-curvature concave, and M3 and M4 are flat mirrors. M1, M2, and M4 are high reflectors at the signal wavelength, and $\mathrm{M} 3$ is an $R \simeq 72 \%$ (measured) output coupler. The PP KTP crystal is positioned at the intracavity focus between M1 and M2. All beams are polarized along the $z$ axis of the crystal to utilize the large $d_{33}$ nonlinear coefficient of KTP. The pump beam is focused with a lens (L) of focal length $75 \mathrm{~mm}$ and enters the cavity through M1, which has hightransmission coatings for this purpose. The lowestorder transverse mode of the cavity has a $48-\mu \mathrm{m}$ waist diameter (calculated), and the focused pump beam has a $70-\mu \mathrm{m}$ waist diameter (measured). M3 is placed upon an open-loop piezocontrolled translation stage with the purpose of adjusting the cavity length for synchronizing the pump and signal pulses. The idler beam exits the cavity through M2, which is transparent $(R<15 \%)$ at this wavelength. Various visible beams resulting from non-phase-matched interactions (frequency-doubled pump and signal, sum of pump with signal and idler) exit the cavity through M2.

The threshold of the OPO is $150 \mathrm{~mW}$ (measured pump power before the lens). At an input pump power of $650 \mathrm{~mW}$ the maximum signal output from M3 is $140 \mathrm{~mW}$, corresponding to a $22 \%$ signal power conversion efficiency and a quantum-limited performance ${ }^{4}$ (photon conversion efficiency) of $34 \%$. At this point the pump depletion is 69\%. Figure 2 shows the signal power conversion efficiency and pump depletion as functions of the input pump power.

Reflection losses incurred by the signal and the pump beams at the uncoated crystal surfaces are the most important factors in limiting the performance of this OPO. The total loss experienced by the pump at the input (L, M1, and the first crystal surface) is $10 \%$. For the resonant signal beam, surface reflection losses per pass through the crystal are $16 \%$. Considering that this loss is comparable with that from output coupling, it is expected to play a significant role in increasing the threshold and decreasing the conversion efficiency. We experimented with output couplers of various reflectivities and found that the lowest reflectivity available to us $(R \simeq 72 \%$ at $1200 \mathrm{~nm})$ provides the highest conversion efficiency. This and the high pump depletion value suggest that the parametric gain in the PP KTP crystal is very high.

We can tune the output of the PP KTP OPO over a 200-nm-wide wavelength range by changing the cavity length. This effect occurs because the cavity roundtrip time for the signal pulse depends on wavelength through the contribution of the group velocity inside the crystal. ${ }^{10}$ Detuning the cavity length by moving one of the cavity mirrors (M3) changes the wavelength of the signal pulses that are synchronous with the pump pulses.

Figure 3 shows the measured signal wavelength as a function of cavity length detuning for a fixed pump wavelength of $758 \mathrm{~nm}$. The theoretical tuning curve, based on the calculated cavity length dependence of the signal wavelength, is in excellent agreement with the data. The corresponding idler tuning range is 2000$3000 \mathrm{~nm}$. Figure 3 also shows the output power variation as we tune the OPO by changing the cavity length. We use the location of the maximum output power to define the zero detuning point for the cavity length. The variation of output power with cavity length detuning is influenced by many factors, such as the wavelength dependence of the output coupler reflectivity, the bandwidth of the cavity mirrors, and the group-velocity mismatch dependence of the parametric gain. ${ }^{11}$ The output coupler reflectivity changes

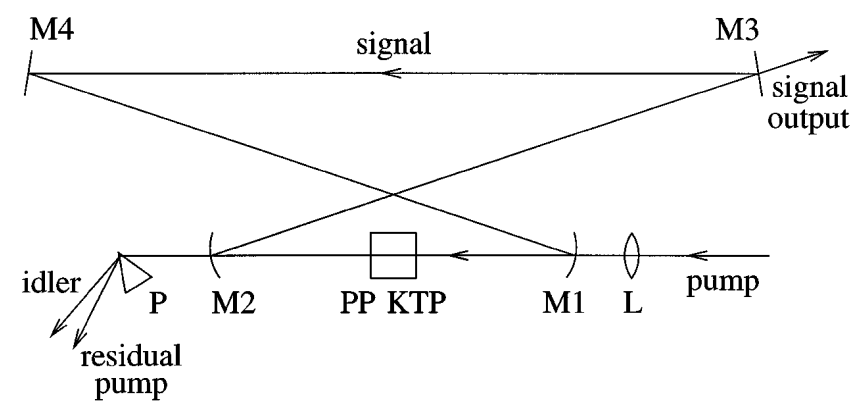

Fig. 1. Schematic of the PP KTP OPO. The pump beam is provided by a mode-locked Ti:sapphire laser.

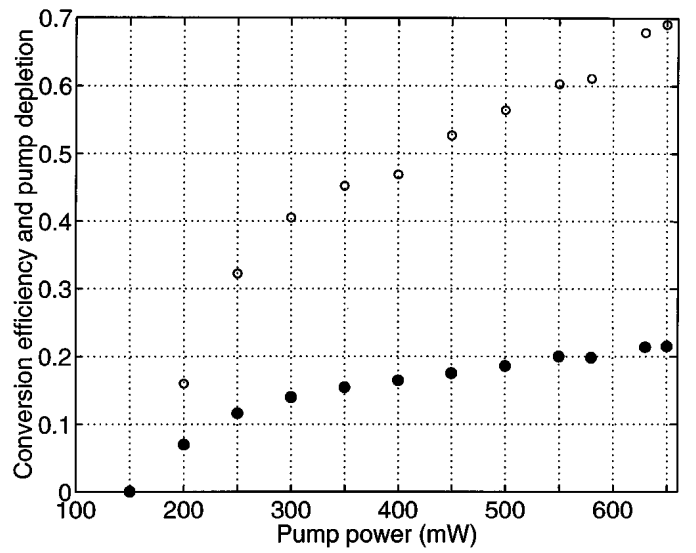

Fig. 2. Conversion efficiency (filled circles) and pump depletion (open circles) versus pump power.

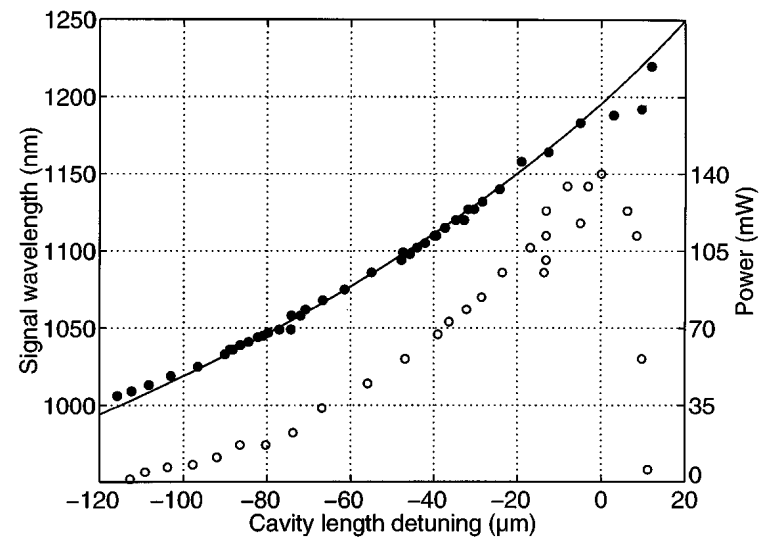

Fig. 3. Measured OPO output wavelength (filled circles) and power (open circles) versus cavity length detuning. The solid curve is a calculated theoretical curve based on equating the wavelength-dependent signal pulse round-trip time to the pump pulse period. 

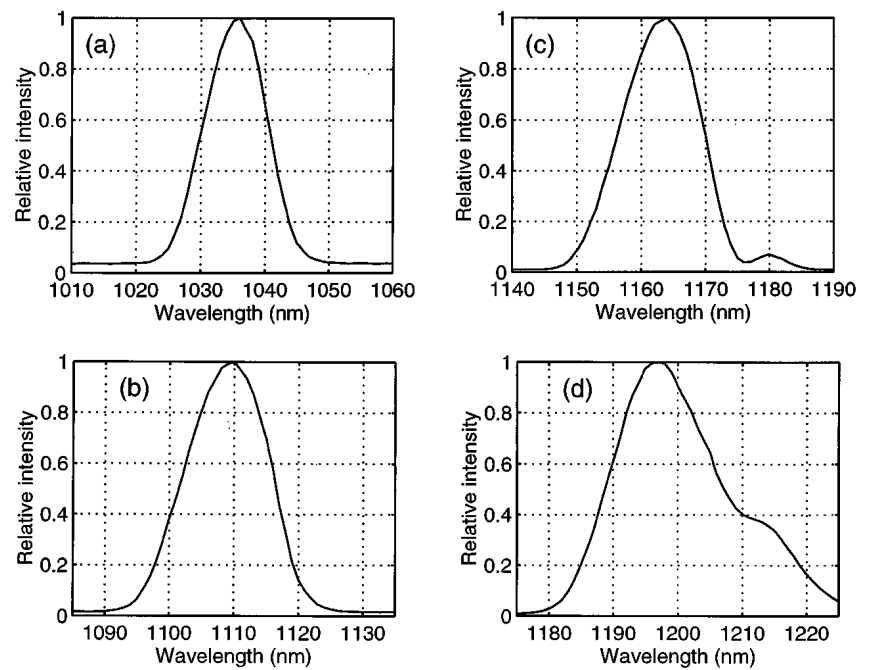

Fig. 4. Spectra of the signal output for cavity detunings of (a) $-88 \mu \mathrm{m}$, (b) $-40 \mu \mathrm{m}$, (c) $-12 \mu \mathrm{m}$, and (d) $0 \mu \mathrm{m}$.

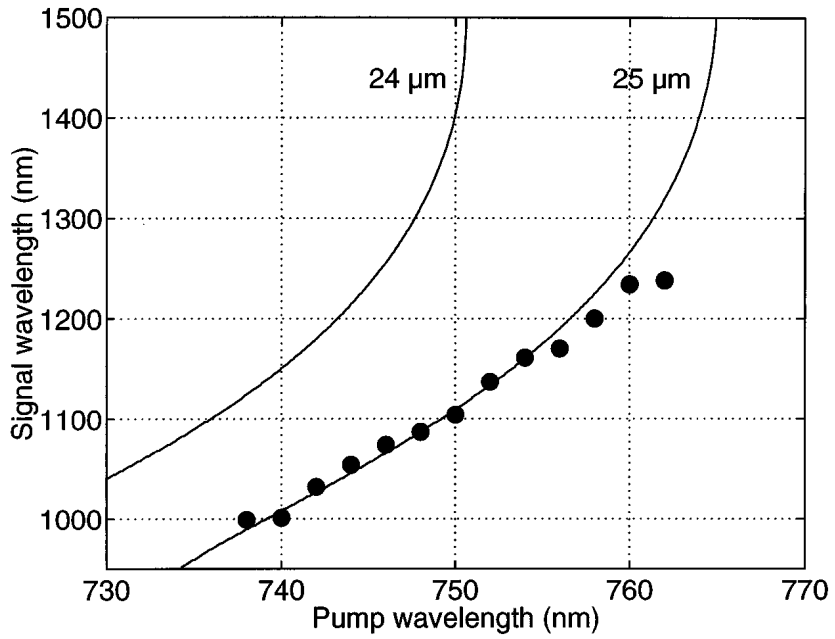

Fig. 5. Measured signal wavelength (filled circles) versus pump wavelength, and calculated theoretical phasematching curves for 24 - and $25-\mu \mathrm{m}$ period gratings.

from $\sim 0.72$ to $\sim 0.91$ within the tuning range. Other cavity mirrors become poor reflectors above $1250 \mathrm{~nm}$. Even though group-velocity mismatch between the signal and the pump pulses limits the parametric interaction length to less than $1 \mathrm{~mm}$, the much longer physical length of the crystal allows for cavity tuning of the signal wavelength.

Figure 4 shows the spectrum of the signal output beam for four values of cavity detuning. The spectra exhibit double-peaked or asymmetric behavior or both because of self-phase modulation and groupvelocity dispersion, a common occurrence in femtosecond OPO's, in which intracavity dispersion is not controlled with prisms. ${ }^{11,12}$ The autocorrelation width of the signal pulses is $330 \mathrm{fs}$. This pulse broadening is due to group-velocity mismatch between the pulses in the crystal. ${ }^{11,12}$ The beam profile of the signal output is measured with a beam profiler to be nearly Gaussian.

It is also possible to tune the PP KTP OPO by varying the wavelength of the pump beam. Experimentally, we were able to demonstrate tunability in the 1000-1235-nm range, being limited by the reflectivity bandwidth of our mirrors and reflection losses from the crystal surfaces. Figure 5 shows the experimental pump tuning data together with calculated curves for 24- and $25-\mu \mathrm{m}$ period domain gratings. We attribute the deviation of the data from the calculated $24-\mu \mathrm{m}$ curve partly to the uncertainty in the orientation of the grating and partly to the inaccuracy of the dispersion relations for KTP. ${ }^{13}$

In conclusion, we have demonstrated a Ti:sapphirelaser-pumped femtosecond PP KTP OPO operating in the 1000-1235-nm wavelength range. The conversion efficiency of the OPO is high despite the presence of high cavity losses other than output coupling. We achieved a wide tunability range by changing the cavity length and the pump wavelength. In particular, cavity length tuning promises to be a powerful technique for obtaining rapid tunability in femtosecond OPO's.

This research was supported in part by the Turkish Scientific and Technical Research Council (Tubitak) under grant EEEAG-118.

\section{References}

1. R. L. Byer and A. Piskarskas, eds., feature on optical parametric oscillators and amplifiers, J. Opt. Soc. Am. B 10, 1656-1791, 2148-2243 (1993); W. R. Bosenberg and R. C. Eckhardt, eds., feature on optical parametric devices, J. Opt. Soc. Am. B 12, 2084-2320 (1995).

2. M. Yamada, N. Nada, M. Saitoh, and K. Watanabe, Appl. Phys. Lett. 62, 435 (1993).

3. L. E. Myers, R. C. Eckardt, M. M. Fejer, R. L. Byer, W. R. Bosenberg, and J. W. Pierce, J. Opt. Soc. Am. B 12, 2102 (1995).

4. W. R. Bosenberg, A. Drobshoff, J. I. Alexander, L. E. Myers, and R. L. Byer, Opt. Lett. 21, 1336 (1996).

5. S. D. Butterworth, P. G. R. Smith, and D. C. Hanna, Opt. Lett. 22, 618 (1997).

6. M. Taya, M. C. Bashaw, and M. M. Fejer, Opt. Lett. 21, 857 (1996).

7. Q. Chen and W. P. Risk, Electron. Lett. 30, 1516 (1994).

8. M. Oron, M. Katz, D. Eger, G. Rosenman, and A. Skliar, Electron. Lett. 33, 807 (1997).

9. H. Karlsson and F. Laurell, in Conference on Lasers and Electro-Optics, Vol. 11 of 1997 OSA Technical Digest Series (Optical Society of America, Washington, D.C., 1997), p. 15.

10. D. C. Edelstein, E. S. Wachman, and C. L. Tang, Appl. Phys. Lett. 54, 1728 (1989).

11. A. Haché, G. R. Allan, and H. M. van Driel, J. Opt. Soc. Am. B 12, 2209 (1995).

12. J. M. Dudley, D. T. Reid, M. Ebrahimzadeh, and W. Sibbett, Opt. Commun. 104, 419 (1994).

13. J. D. Bierlein and H. Vanherzeele, J. Opt. Soc. Am. B 6, 622 (1989). 